\title{
Reflections on Engineering Design Education
}

\author{
Ralph O. Buchal \\ Department of Mechanical \& Materials Engineering \\ The University of Western Ontario \\ London, Ontario, Canada \\ rbuchal@eng.uwo.ca
}

\begin{abstract}
Engineering design has received increasing attention in the undergraduate engineering curriculum, and much progress is being made. However, deficiencies are still observed in many skills required to be a successful design engineer, including: design methodology, project management skills, engineering analysis and validation in design, engineering graphics, practical skills, and reflection. Important factors affecting these outcomes include clear understanding of the design process, mentorship and direction from engineering advisors, increased opportunity for practical experience, and clear expectations.
\end{abstract}

\section{Introduction}

This paper is a collection of personal observations, reflections and recommendations resulting from many years of teaching engineering design, and supervising student design projects.

\section{The Engineering Design Process}

Historically, engineering design has been considered a skill that is learned by experience and practice. In recent decades, design researchers and practitioners have worked to develop a more systematic approach to design. Today, virtually all engineering schools teach the engineering design process, and many practicing engineers and companies have adopted formal design processes as well. Many excellent textbooks have appeared to guide students and practitioners $[1,2,3,4$, $5,6]$.

While there is general agreement on the usefulness of adopting a systematic design process, there is no agreement on a single ideal process. Design remains an imprecise activity, and the purpose of a systematic approach is to provide a heuristic framework and guidelines rather than a rigid sequence of steps. Most designers agree that the design process involves several stages: Planning and Task Clarification; Conceptual Design; Embodiment Design; and Detail Design. A design project must pass through all of these stages, more or less in order. However, the boundaries between stages can be fuzzy, and backtracking and iteration are expected.

Adoption of a systematic approach helps prevent many of the common errors of student and novice designers, including:

- Not defining the problem, or solving the wrong problem

- Pursuing the first idea that occurs, and not considering alternatives

- Not documenting and justifying design decisions

Experienced designers already internalize many of the steps of the process, but Pahl et al. [1] argue that even experts can benefit from a more systematic approach to design. Better results can be obtained, and in many cases, little if any extra time is required.

\section{Project Management}

Project management involves identification and allocation of resources (personnel, equipment, funding, etc.), and scheduling of tasks to achieve desired milestones. At the beginning of a project, designers usually lack the knowledge or information to accurately estimate the resources required, or to prepare a detailed schedule. Experienced engineers can 
rely on knowledge of similar past projects, but students lack this frame of reference. Student estimates of time and resources required can be very inaccurate. Students tend to greatly underestimate the time and resources required to complete a project, especially for the embodiment and detail design stages. It is important for students to learn that the design project is far from finished at the end of the conceptual design stage. Unfortunately, many student projects do not get past this stage.

\section{Emerging Technologies and Innovation}

Many design courses and methodologies rely on illdefined methods like brainstorming or flashes of inspiration to generate innovative design concepts. In well-explored design domains, these methods are unlikely to produce innovative designs, especially for novice designers. Furthermore, students typically lack the engineering knowledge and background to identify and assess the feasibility of well-known alternatives and prior art. Hence, the majority of student "innovations" are impractical or impossible.

Design innovation requires a broad knowledge of existing and emerging technologies and principles from multiple disciplines. Experienced engineers build up a storehouse of knowledge that allows them to generate many design concepts quickly and effortlessly. Students and novice designers must build up this knowledge through research and experience. Furthermore, an explicit process of generating and evaluating alternatives should be followed and documented.

\section{Conceptual versus Embodiment Design}

In many design courses, emphasis is placed on one or two of the stages of the design process, while the other stages are considered superficially if at all.

For example, many introductory design courses focus on conceptual design, with relatively little attention to embodiment and detail design. These courses focus on qualitative methods like brainstorming, Quality Function Deployment (QFD), functional decomposition, decision matrices, etc. Design projects emphasize creativity rather than realism. Concepts are represented by rough sketches, and prototypes are simple. Generally little analysis or testing is done.
The resulting designs are generally only rough concepts, not finished designs. Students often do not appreciate this fact. They use tools like decision matrices to justify a weak design concept because it produces a higher numerical score than other weak concepts. However, students usually cannot justify the criteria, weights and ratings they have used to evaluate the alternatives. If drawings or CAD models are produced, they are often inadequate to allow concepts to be properly evaluated, or for prototypes to be built.

Alternatively, many mechanical component design courses place emphasis on embodiment and detail design. The focus is on modeling and analysis rather than generation and evaluation of alternatives. These courses typically use the "Shigley" approach to design, in reference to the very widely used textbooks by Shigley [7, 8] Many of these courses are based on well-defined end-of-chapter problems rather than openended design projects. An outcome of a design project in these courses may be a set of detailed working drawings and calculations of a specified mechanical system, with no consideration of possible alternative solutions.

All stages of the design process are essential, and it is important that none are skipped. Students should experience the entire process, including defining the problem, generating and evaluating alternatives, and developing concepts to the point where they are validated and can be built or tested.

\section{Engineering Analysis and Validation}

A goal of design is to conceive of a technical system to perform a desired set of functions, and prepare detailed instructions to allow the technical system to be built. Tinkerers and backyard inventors do this through experience and trial-and-error. Engineers, on the other hand, use knowledge of engineering science to understand, analyze and predict the performance of technical systems before they are built. The majority of the undergraduate engineering curriculum is devoted to building the necessary foundation in engineering science.

Many students have difficulty establishing connections between their various engineering science courses and practical design problems. Many still have fundamental misunderstandings of basic engineering concepts, and have difficulty performing appropriate and correct engineering analysis. Often students resort to qualitative reasoning when quantitative analysis could be done. For example, they might refer to their design 
as being "lightweight" or "strong" without quantifying these in engineering terms.

\subsection{Mathematical Modeling and Estimation}

Mathematical modeling and analysis are fundamental to engineering design. At the early stages of design, these models can be very simple. The purpose is to provide rough estimates rather than precise results. These "back of the envelope" calculations are used to establish orders of magnitude estimates based on incomplete and approximate information. As knowledge of the design increases, more accurate models and analyses are used.

Many students are unable to develop a simple mathematical model, or to perform a rough calculation. They are unsure of how to proceed if some information is uncertain or unknown. Many students do not understand significant figures, and carry out all calculations to high precision. If they are asked to estimate the circumference of a tree that is about $1 \mathrm{~m}$ in diameter, the answer is invariably $3.1415927 \mathrm{~m}$, with the number of significant figures dependent on the number of digits displayed on their calculator.

\subsection{Computer Modeling and Simulation}

Computer modeling and simulation are very powerful techniques, especially in the later stages of design when more accurate and detailed analyses are appropriate. Modern CAD and CAE software packages permit the modeling and analysis of "virtual prototypes", greatly reducing the need to build and test physical prototypes.

As with any tool, the potential for misuse is great. Current integrated CAD/CAE packages allow students to conduct FEA analysis of a CAD model with just a few clicks of the mouse. As a result, students often use FEA inappropriately as a substitute for simple calculations, and ascribe too much weight to the results. Often FEA is used when the geometry is still preliminary and conceptual, and the loads and restraints are nothing but guesses. The results are then presented to many significant figures.

\subsection{Testing and Experimentation}

$\mathrm{CAD}$ and $\mathrm{CAE}$ have reduced but not eliminated the need for testing and experimentation. In real design situations, the values of parameters are not given as they are in homework problems. In some cases, these parameter values can be obtained from handbooks and other sources. In other cases, they can be determined experimentally. Often, empirical models of system behavior must be developed through experimentation. Finally, design prototypes are built and tested to validate analysis and simulation results, and to confirm that the design will perform as predicted.

Most student lab experience is composed of conducting predefined experiments designed by others. Students lack confidence to design and conduct an experiment of their own in the context of a design project. Often the required experiment is very simple. Examples of parameters that might need to be determined experimentally include rolling resistance, coefficient of friction, efficiency, mass, deflection, etc.

\section{$7 \quad$ The Role of Prototypes}

According to many definitions of design, the design process ends with a complete set of instructions to produce the artifact. Prototypes are used at every stage of design process. Prototypes can take many forms, from simple models made of cardboard to fullyfunctional systems. Prototypes are used to communicate and test ideas, to aid in visualization, to test and validate the performance of components and subsystems, to test overall function using different materials or simplified design, to test function using the final design and materials but different manufacturing methods, etc.

Most design project courses require some form of prototype as a project deliverable, but often the meaning of "prototype" is not defined or discussed. It is important that the students and instructors share a clear understanding of the purpose and types of design prototypes; otherwise, there is a risk of incompatible expectations. Everyone needs to understand that most students do not have the skills or resources to construct professional-quality functional prototypes of anything but the most trivial design artifact. Rough prototypes made of scrap material and simple tools are useful in design, but these prototypes are not usually expected to be fully functional. Consequently, the quality of the design cannot be judged solely by the functionality of a student-built prototype.

It is very valuable for students to work with technicians to have functional prototypes built from drawings and CAD models. This provides an opportunity for students to see the difference in the quality of different prototypes, and to appreciate the complementary skills of professional technicians. However, this requires significant resources. 


\section{CAD and Engineering Graphics}

Engineering graphics is being de-emphasized in many engineering curricula. As a result, many students have little experience or training in engineering sketching or basic drawing standards and practices. Their understanding of dimensioning and tolerancing is weak. As a result, their sketches and drawings are often too vague, conceptual and incomplete to allow a part to be made.

Feature-based solid modeling CAD is replacing drawing as a design tool. Students enjoy CAD, and many become very good at using the software. However, the resulting models are often constructed with little thought about the details of how the parts and assembly will be made. CAD models are usually constructed using feature operations that do not correspond directly to manufacturing processes, and often the resulting part is difficult or impossible to manufacture. Since modeling complex geometry is so easy, students do not think of using simple standard shapes, perhaps welded or bolted together, to achieve the same function. Most student designs require CNC machining from solid billet.

Also, many CAD models are nothing more than 3D conceptual sketches. The models lack the necessary details to allow them to be made. Missing details can include: bolt holes, brackets, threaded holes, keyways, grooves and retaining rings, bearings and bushings, etc.

\section{The Role of Faculty Advisors}

Engineering design cannot be learned solely from books, but must be learned through experience and apprenticeship. The role of faculty advisors is critical. If students do not get enough guidance, many flounder and learn very little. If the faculty advisor manages the project too closely, students may end up performing well-defined tasks assigned by the advisor rather than managing the project themselves. Ideally, the faculty advisor serves as a mentor to guide and provide feedback and advice on a regular basis, while giving the students freedom to manage the project.

Effective faculty mentorship can be a challenge if faculty members lack either interest or design experience. This can be mitigated to some extent by the adoption of very clear guidelines and expectations, and by involving other advisors including industry supervisors and university technicians.

\section{The Value of Failures}

Design failures can be a better teacher than successes, but only if the designer reflects on the cause of the failure. Students are often too quick to blame failures on faulty material, parts or workmanship, without investigating the true cause. Even when the cause is correctly identified, this is not always documented or shared with other members of the team. Learning from failures is particularly important for student design teams like Formula SAE, SAE Baja, etc.

\section{Practical Skills}

The emphasis of the engineering curriculum is on engineering science, rather than engineering practice and design. As a result, many students do not develop the level of practical knowledge and experience expected from graduate engineers.

Students have a weak understanding of common manufacturing processes, and little if any practical experience. As a result, they design parts with little thought as to how they would be made. Often the parts are difficult or impossible to make. Designs include features like square holes, very deep small diameter holes, etc. Also, students do not consider alternative methods of manufacturing such as welding standard shapes versus machining from billet.

Students have very little practical knowledge of fastening and joining methods, standard mechanical components, standard design solutions, etc. Their CAD assembly models often have no provisions for joining, connecting and supporting parts in the assembly.

Many students are unaware of the existence of engineering standards, found in engineering handbooks. They do not know how to select an appropriate bearing or gear from a catalog. They do not realize that there are standard methods for mounting bearings and gears.

It is unclear how to best develop the required practical skills in engineering students. These skills are best developed through experience and mentorship, ideally by repeated opportunities to experience the designbuild-test cycle. Prototype building and testing are expensive and resource-intensive, so schools are often forced to cut corners. Students to not gain the necessary skills through simple paper or "toy" design projects. 
The main opportunity available to students is participation in student design competitions like Formula SAE and solar car competitions. Participation in these projects can develop many skills not addressed by the formal engineering curriculum $[9,10]$.

It is also very useful to have students work with professional technicians to have their designs made from drawings and CAD models. They receive detailed feedback and advice on their designs, with suggestions for changes to improve manufacturability.

Much can be learned through reverse engineering and case studies, if it is done in a structured manner. To maximize the benefit, an existing design artifact could be disassembled and fully documented in terms of function, structure, components, analysis, materials and manufacturing methods.

Another innovation being explored by the Department of Mechanical and Materials Engineering at the Univerity of Western Ontario is a partnership with Fanshawe College to allow Mechanical Engineering students to complete an accelerated Manufacturing Engineering Diploma to complement their engineering education.

\section{Expectations and Evaluation Criteria}

Student design projects are often evaluated by faculty advisors with various backgrounds, and each faculty advisor has different personal criteria for evaluating a design project. Furthermore, the projects themselves can be quite different. To ensure consistent assessment, it is important to develop and communicate clear evaluation criteria and expectations to both students and faculty advisors. The evaluation criteria should be suitable for any project, and should be based on accepted design practices.

Innovative or creative design projects may emphasize problem definition and conceptual design, with limited embodiment and detail design. Analysis might involve simple mathematical models, with incomplete information and many estimates. Prototyping might be limited to $\mathrm{CAD}$ models, computer simulations, rapid prototypes, and prototypes of subsystems. An example might be the design of a new type of personal transportation system.

Simple or routine design projects might emphasize embodiment and detail design. Detailed mathematical modeling, computer simulation and testing would be expected. Detailed CAD models along with working drawings and parts lists would be a deliverable. A fully functional prototype might be built using the final materials, but different manufacturing processes. For well-understood applications, a complete set of working drawings to allow the design to be built might be sufficient.

Typically, a significant part of the overall project evaluation is based on what is documented in the final report and supporting information. At Western, we are continually evolving and clarifying a set of clear expectations and evaluation criteria for design reports as described in the following sections.

\subsection{Problem Definition}

The report must clearly and correctly identify and define the problem, requirements, and design constraints.

\subsection{Background Research}

The report should demonstrate sufficient research into the background of the problem, existing state-of-theart, and emerging technologies relevant to the project. The report should also demonstrate research into areas of engineering science and practice relevant to the project. Examples include: theoretical models and techniques, analysis methods, experimental methods, sensors, actuators, manufacturing methods, standards, mechanical components, software tools, etc.

\subsection{Design Methodology}

The report must demonstrate the use of appropriate engineering design methodology. Multiple alternatives must have been considered and evaluated before selecting a final design or solution. Decisions must be properly documented and justified. Non-technical issues such as environmental impact, safety, legal requirements, etc. must be considered as appropriate.

\subsection{Engineering Validation}

An appropriate understanding and application of engineering principles and concepts must be demonstrated. Design decisions, evaluations and trade-offs must be properly justified using mathematical modeling, computer simulation, experimentation and prototype testing as appropriate.

\subsection{Design Documentation}

Designs and solutions must be fully documented with working drawings, bills of materials, cost analysis, etc. as appropriate. There should be sufficiently detailed documentation to permit the project to be duplicated or continued by others. 


\subsection{Technical Writing and General Organization}

The report should be written clearly and concisely. It must be carefully proofread, and should contain no grammatical, typographic or spelling errors. The report should be logically organized.

\subsection{Formatting}

The report should be properly formatted, with a cover page, table of contents, list of figures and tables as appropriate. All references must be correctly cited using a standard reference style, and the reference list must be complete. All figures must be labeled and numbered, and referred to in the text. Appendices should be used appropriately for supplemental material, but key information must appear in the body of the report.

\subsection{Supporting Digital Information}

In addition to a printed report, all relevant digital files must be submitted on a DVD or equivalent medium. These files include: all reports and presentations, spreadsheets, multimedia files, CAD files, etc.

\section{Conclusions}

This paper presents personal observations and reflections on engineering design education approaches and outcomes at the University of Western Ontario. The issues discussed in this paper are not unique to Western, but are relevant to most engineering schools. Identifying and addressing weaknesses in educational outcomes remains an ongoing challenge for engineering design educators.

\section{References}

[1] G. Pahl, W. Beitz, J. Feldhusen, and K.H. Grote, Engineering Design: A Systematic Approach, Third Edition, Springer 2007.

[2] W. E. Eder, and S. Hosnedl, Design Engineering: A Manual for Enhanced Creativitiy, CRC Press, 2008.

[3] D. G. Ullman, The Mechanical Design Process, Third Edition, McGraw-Hill, 2003.

[4] K. Otto, and K. Wood, Product Design: Techniques in Reverse Engineering and New Product Development, Prentice Hall, 2001.

[5] R. J. Eggert, Engineering Design, Pearson, 2005.

[6] G. E. Dieter, Engineering Design: A Materials and Processing Approach, Third Edition, McGraw Hill, 2000.
[7] J. E. Shigley, Mechanical Engineering Design, Third Edition, McGraw-Hill, 1977.

[8] R. Budynas, and J. K. Nisbett, Shigley's Mechanical Engineering Design, Eighth Edition, McGraw-Hill, 2006.

[9] R. O. Buchal, "The Educational Value of Student Design Competitions", Proceedings of The Inaugural CDEN Design Conference, Montreal, July 2004.

[10] R. O. Buchal; I. Atcha; A. Da Rocha; R. Jelenik; P. Kriznic; "Student Design Teams at UWO: a Case Study", Proceedings of the Second CDEN International Conference on Design Education, Innovation, and Practice, Kananaskis, Alberta, July 2005 